MATHEMATICS OF COMPUTATION

Volume 69, Number 231, Pages 1207-1212

S 0025-5718(99)01118-7

Article electronically published on February 19, 1999

\title{
ON A UNIT GROUP GENERATED BY SPECIAL VALUES OF SIEGEL MODULAR FUNCTIONS
}

\author{
T. FUKUDA AND K. KOMATSU
}

\begin{abstract}
There has been important progress in constructing units and $S$ units associated to curves of genus 2 or 3 . These approaches are based mainly on the consideration of properties of Jacobian varieties associated to hyperelliptic curves of genus 2 or 3 . In this paper, we construct a unit group of the ray class field $k_{6}$ of $\mathbb{Q}(\exp (2 \pi i / 5))$ modulo 6 with full rank by special values of Siegel modular functions and circular units. We note that $k_{6}=\mathbb{Q}(\exp (2 \pi i / 15), \sqrt[5]{-24})$. Our construction of units is number theoretic, and closely based on Shimura's work describing explicitly the Galois actions on the special values of theta functions.
\end{abstract}

\section{THEOREM}

We begin by explaining the notation. We denote as usual by $\mathbb{Z}, \mathbb{Q}, \mathbb{R}$ and $\mathbb{C}$ the ring of rational integers and the fields of rational numbers, real numbers and complex numbers, respectively. For a positive integer $n, \mathbb{Z}^{n}, \mathbb{Q}^{n}$, etc. denote the module or vector space of $n$-dimensional column vectors with components in $\mathbb{Z}, \mathbb{Q}$, etc. If $Y$ is an associative ring with identity element, then $Y^{\times}$denotes the group of all invertible elements of $Y$, and $M_{n}(Y)$ the ring of all matrices of degree $n$ with components in $Y$; the identity element of $M_{n}(Y)$ is denoted by $I_{n}$. We write $G L_{n}(Y)=M_{n}(Y)^{\times}$. The transpose of a matrix $\alpha$ is denoted by ${ }^{t} \alpha$. For elements $g_{1}, \cdots, g_{r}$ of a group $G$, we denote by $\left\langle g_{1}, \cdots, g_{r}\right\rangle$ the subgroup of $G$ generated by $g_{1}, \cdots, g_{r}$.

For a finite algebraic extension $K$ of $k$, we denote by $[K: k]$ the degree of $K$ over $k$, and if $K$ is a Galois extension of $k$, we denote by $G(K / k)$ the Galois group of $K$ over $k$.

Let $\mathfrak{S}_{2}$ be the set of all complex symmetric matrices of degree 2 with positive definite imaginary parts. For $u \in \mathbb{C}^{2}, z \in \mathfrak{S}_{2}$ and $r, s \in \mathbb{R}^{2}$, put as usual

$$
\Theta(u, z ; r, s)=\sum_{x \in \mathbb{Z}^{2}} e\left(\frac{1}{2}{ }^{t}(x+r) z(x+r)+{ }^{t}(x+r)(u+s)\right),
$$

where $e(\xi)=\exp (2 \pi i \xi)$ for $\xi \in \mathbb{C}$. Moreover we put

$$
\Phi\left(z ; r, s ; r_{1}, s_{1}\right)=\frac{2 \Theta(0, z ; r, s)}{\Theta\left(0, z ; r_{1}, s_{1}\right)}
$$

for $r_{1}, s_{1} \in \mathbb{R}^{2}$.

Received by the editor October 16, 1997 and, in revised form, August 14, 1998.

1991 Mathematics Subject Classification. Primary 11G15, 11R27, 11Y40.

Key words and phrases. Siegel modular functions, unit groups, computation. 
Let $\Gamma_{1}=S_{p}(2, \mathbb{Z})=\left\{\left.\alpha \in G L_{4}(\mathbb{Z})\right|^{t} \alpha J \alpha=J\right\}$, where

$$
J=\left(\begin{array}{rrrr}
0 & 0 & -1 & 0 \\
0 & 0 & 0 & -1 \\
1 & 0 & 0 & 0 \\
0 & 1 & 0 & 0
\end{array}\right)
$$

For every positive integer $N$, we put $\Gamma_{N}=\left\{\alpha \in \Gamma_{1} \mid \alpha \equiv I_{4}\left(\bmod N M_{4}(\mathbb{Z})\right)\right\}$. We let every element $\alpha=\left(\begin{array}{ll}A & B \\ C & D\end{array}\right)$ act on $\mathfrak{S}_{2}$ by $\alpha(z)=(A z+B)(C z+D)^{-1}$ for $z \in \mathfrak{S}_{2}$.

Let $v$ be a non-zero integer and $\alpha$ a matrix in $M_{4}(\mathbb{Z})$ with ${ }^{t} \alpha J \alpha=v J$. We suppose that the determinant of $\alpha$ is $v^{2}$ and that $v$ is prime to $2 N$. Then it is well known that there exists a matrix $\beta_{\alpha}$ in $\Gamma_{1}$ with

$$
\alpha \equiv\left(\begin{array}{cc}
I_{2} & 0 \\
0 & v I_{2}
\end{array}\right) \beta_{\alpha} \quad\left(\bmod 2 N^{2}\right) .
$$

Let $r, s, r_{1}, s_{1} \in \frac{1}{N} \mathbb{Z}^{2}$. Then $\Phi\left(z ; r, s ; r_{1}, s_{1}\right)$ is a Siegel modular function of level $2 N^{2}$ (cf. [5], Proposition 1.7). We let $\alpha$ act on $\Phi\left(z ; r, s ; r_{1}, s_{1}\right)$ by

$$
\Phi^{\alpha}\left(z ; r, s ; r_{1}, s_{1}\right)=\Phi\left(\beta_{\alpha}(z) ; r, v s ; r_{1}, v s_{1}\right) .
$$

We note that $\Phi^{\alpha}$ is also a Siegel modular function of level $2 N^{2}$.

Now, we put $\zeta_{n}=\exp (2 \pi i / n)$ for a positive integer $n$ and $k=\mathbb{Q}\left(\zeta_{5}\right)$. In what follows, we concentrate our attention on the case $n=5$. So we write for simplicity $\zeta=\zeta_{5}$. Let $\mathfrak{O}_{k}$ be the integer ring of $k$ and $\sigma$ the element of the Galois group $G(k / \mathbb{Q})$ defined by $\zeta^{\sigma}=\zeta^{2}$. For the 2 -dimensional complex vector space $\mathbb{C}^{2}$, we put $L=\left\{\left(\begin{array}{c}\xi \\ \xi^{\sigma}\end{array}\right) \in \mathbb{C}^{2} \mid \xi \in \mathfrak{O}_{k}\right\}$. Then $L$ is a lattice in $\mathbb{C}^{2}$. We put $\alpha=\left(\zeta-\zeta^{4}\right) / 5$ and define a Riemann form $E$ on the complex torus $\mathbb{C}^{2} / L$ as follows:

$$
E\left(\left(\begin{array}{l}
u_{1} \\
u_{2}
\end{array}\right),\left(\begin{array}{l}
v_{1} \\
v_{2}
\end{array}\right)\right)=\alpha\left(u_{1} \bar{v}_{1}-\bar{u}_{1} v_{1}\right)+\alpha^{\sigma}\left(u_{2} \bar{v}_{2}-\bar{u}_{2} v_{2}\right)
$$

for $u_{i}, v_{i} \in \mathbb{C}$. Moreover, for $\boldsymbol{\omega}_{1}=\left(\begin{array}{c}-\zeta \\ -\zeta^{2}\end{array}\right), \boldsymbol{\omega}_{2}=\left(\begin{array}{c}\zeta^{4} \\ \zeta^{3}\end{array}\right), \boldsymbol{\omega}_{3}=\left(\begin{array}{c}\zeta^{2}+\zeta^{4} \\ \zeta^{4}+\zeta^{3}\end{array}\right)$ and $\boldsymbol{\omega}_{4}=$ $\left(\begin{array}{c}\zeta^{3} \\ \zeta\end{array}\right)$, we can easily see that $\left\{\boldsymbol{\omega}_{1}, \boldsymbol{\omega}_{2}, \boldsymbol{\omega}_{3}, \boldsymbol{\omega}_{4}\right\}$ is a free basis of $L$ over $\mathbb{Z}$ and $\left(E\left(\boldsymbol{\omega}_{i}, \boldsymbol{\omega}_{j}\right)\right)_{i, j=1,2,3,4}=J$. Hence we see that

$$
\begin{aligned}
z_{0} & =\left(\begin{array}{cc}
\zeta^{2}+\zeta^{4} & \zeta^{3} \\
\zeta^{4}+\zeta^{3} & \zeta
\end{array}\right)^{-1}\left(\begin{array}{cc}
-\zeta & \zeta^{4} \\
-\zeta^{2} & \zeta^{3}
\end{array}\right) \\
& =\frac{1}{5}\left(\begin{array}{cc}
2+\zeta-\zeta^{3}-2 \zeta^{4} & 2-\zeta+\zeta^{2}-2 \zeta^{3} \\
2-\zeta+\zeta^{2}-2 \zeta^{3} & \zeta+2 \zeta^{2}-2 \zeta^{3}-\zeta^{4}
\end{array}\right)
\end{aligned}
$$

is a CM-point of $\mathfrak{S}_{2}$ corresponding to the polarized abelian variety $\left(\mathbb{C}^{2} / L, E\right)$.

Now, let $\omega$ be an element of $\mathfrak{O}_{k}$. We denote by

$$
R(\omega)=\left(\begin{array}{cccc}
a_{11} & a_{12} & a_{13} & a_{14} \\
a_{21} & a_{22} & a_{23} & a_{24} \\
a_{31} & a_{32} & a_{33} & a_{34} \\
a_{41} & a_{42} & a_{43} & a_{44}
\end{array}\right)
$$

the regular representation of $\omega$ with respect to $\xi_{1}=-\zeta, \xi_{2}=\zeta^{4}, \xi_{3}=\zeta^{2}+\zeta^{4}$, $\xi_{4}=\zeta^{3}$. Namely,

$$
\omega \xi_{i}=\sum_{j=1}^{4} a_{i j} \xi_{j} \quad \text { with } a_{i j} \in \mathbb{Z}
$$


Then there exists an integer $v$ with ${ }^{t} R\left(\omega \omega^{\sigma^{3}}\right) J R\left(\omega \omega^{\sigma^{3}}\right)=v J$, $\operatorname{det} R\left(\omega \omega^{\sigma^{3}}\right)=v^{2}$ and $R\left(\omega \omega^{\sigma^{3}}\right) z_{0}=z_{0}$ (cf. [5] p. 684]). Now we define an endomorphism $\varphi$ of $k^{\times}$by $\varphi(a)=a^{1+\sigma^{3}}$ for $a \in k^{\times}$. We have

$$
R(\varphi(\zeta+2))=R\left((\zeta+2)(\zeta+2)^{\sigma^{3}}\right)=\left(\begin{array}{rrrr}
3 & 0 & -1 & 1 \\
2 & 2 & 0 & -1 \\
3 & -2 & 2 & -1 \\
-2 & 1 & 1 & 4
\end{array}\right) .
$$

The main purpose of this paper is to prove the following theorem:

Theorem. Let $k=\mathbb{Q}(\exp (2 \pi i / 5))$, and let $k_{6}$ be the ray class field of $k$ modulo 6 . We put

$$
\varepsilon_{\nu}=\frac{\Phi^{R\left(\varphi(2+\zeta)^{\nu}\right)}\left(z_{0} ;\left(\begin{array}{l}
\frac{1}{3} \\
0
\end{array}\right),\left(\begin{array}{l}
0 \\
0
\end{array}\right) ;\left(\begin{array}{l}
0 \\
0
\end{array}\right),\left(\begin{array}{l}
0 \\
0
\end{array}\right)\right)}{\Phi^{R\left(\varphi(2+\zeta)^{\nu+2}\right)}\left(z_{0} ;\left(\begin{array}{l}
\frac{1}{3} \\
0
\end{array}\right),\left(\begin{array}{l}
0 \\
0
\end{array}\right) ;\left(\begin{array}{l}
0 \\
0
\end{array}\right),\left(\begin{array}{l}
0 \\
0
\end{array}\right)\right)}
$$

and

$$
\eta_{\nu}=\frac{\Phi^{R\left(\varphi(2+\zeta)^{\nu}\right)}\left(z_{0} ;\left(\begin{array}{c}
\frac{1}{3} \\
\frac{2}{3}
\end{array}\right),\left(\begin{array}{c}
\frac{1}{3} \\
0
\end{array}\right) ;\left(\begin{array}{l}
0 \\
0
\end{array}\right),\left(\begin{array}{l}
0 \\
0
\end{array}\right)\right)}{\Phi^{R\left(\varphi(2+\zeta)^{\nu+2}\right)}\left(z_{0} ;\left(\begin{array}{c}
\frac{1}{3} \\
\frac{2}{3}
\end{array}\right),\left(\begin{array}{c}
\frac{1}{3} \\
0
\end{array}\right) ;\left(\begin{array}{l}
0 \\
0
\end{array}\right),\left(\begin{array}{l}
0 \\
0
\end{array}\right)\right)}
$$

for all $\nu \in \mathbb{Z}$. Moreover we put $\rho_{0}=1-\zeta_{15}, \rho_{1}=1-\zeta_{15}^{7}$ and $\rho_{2}=1-\zeta_{15}^{11}$. Then the subgroup $\left\langle\varepsilon_{0}, \cdots, \varepsilon_{7}, \eta_{0}, \cdots, \eta_{7}, \rho_{0}, \rho_{1}, \rho_{2}\right\rangle$ of $k_{6}^{\times}$is a unit group of $k_{6}$ of free rank 19 .

\section{Proof of the Theorem}

Let $m$ be a positive integer. We put $S_{m}=\left\{a \in k^{\times} \mid a \equiv 1(\bmod m)\right\}$ and $\tilde{S}_{m}=\left\{(a) \mid a \in S_{m}\right\}$, where $(a)$ is the principal ideal of $k$ generated by $a$. Let $U$ be the unit group of $k$ and $k_{m}$ the ray class field of $k$ modulo $m$. Then we have

$$
G\left(k_{18} / k_{6}\right) \cong \tilde{S}_{6} / \tilde{S}_{18} \cong S_{6} U / S_{18} U \cong S_{6} / S_{18}\left(S_{6} \cap U\right)
$$

by class field theory. We put $\omega_{1}=1+6=1+6\left(-\zeta-\zeta^{2}-\zeta^{3}-\zeta^{4}\right), \omega_{2}=$ $1+6\left(\zeta-\zeta^{4}\right), \omega_{3}=1+6\left(\zeta^{2}-\zeta^{3}\right), \omega_{4}=1+6\left(\zeta-\zeta^{2}-\zeta^{3}+\zeta^{4}\right)=1+2 \sqrt{5}$ and $H=S_{18}\left(S_{6} \cap U\right)$. Since $\left\{\zeta, \zeta^{2}, \zeta^{3}, \zeta^{4}\right\}$ is a free basis of $\mathfrak{O}_{k}$ over $\mathbb{Z}$ and $S_{6} / S_{18}$ is mapped isomorphicaly to $\mathfrak{O}_{k} / 3 \mathfrak{O}_{k}$ by the mapping $S_{6} / S_{18} \ni(1+6 \omega) S_{18} \mapsto \omega+$ $3 \mathfrak{O}_{k} \in \mathfrak{O}_{k} / 3 \mathfrak{O}_{k}$, we have $S_{6} / S_{18} \cong(\mathbb{Z} / 3 \mathbb{Z})^{4}$ and $S_{6} / S_{18}=\left\langle(1+6 \zeta) S_{18},\left(1+6 \zeta^{2}\right) S_{18}\right.$, $\left.\left(1+6 \zeta^{3}\right) S_{18},\left(1+6 \zeta^{4}\right) S_{18}\right\rangle$. Hence we have $S_{6} / S_{18}=\left\langle\omega_{1} S_{18}, \omega_{2} S_{18}, \omega_{3} S_{18}, \omega_{4} S_{18}\right\rangle$, because

$$
\operatorname{det}\left(\begin{array}{rrrr}
-1 & -1 & -1 & -1 \\
1 & 0 & 0 & -1 \\
0 & 1 & -1 & 0 \\
1 & -1 & -1 & 1
\end{array}\right)=-8
$$

and $(8,3)=1$, which shows that $S_{6} / H=\left\langle\omega_{1} H, \omega_{2} H, \omega_{3} H, \omega_{4} H\right\rangle$ as $H \supset S_{18}$. We put $\theta_{1}=1+4 \cdot 3, \theta_{2}=1+6\left(\zeta-\zeta^{2}+\zeta^{3}-\zeta^{4}\right), \theta_{3}=1+6\left(\zeta+\zeta^{2}-\zeta^{3}-\zeta^{4}\right)$ and $\theta_{4}=1$, so that we have $\varphi\left(\omega_{i}\right) H=\theta_{i} H$ for $i=1,2,3$, 4. Since $\varphi(H) \subset H$, we can define an endomorphism $\tilde{\varphi}$ of $S_{6} / H$ by $\tilde{\varphi}(a H)=\varphi(a) H$. By a standard argument in linear algebra, we see that $\tilde{\varphi}\left(S_{6} / H\right)=\left\langle\theta_{1} H, \theta_{2} H, \theta_{3} H\right\rangle \cong(\mathbb{Z} / 3 \mathbb{Z})^{3}$. Thus for the class field $K$ of $k$ corresponding to the kernel of $\tilde{\varphi}$, we have $G\left(K / k_{6}\right) \cong(\mathbb{Z} / 3 \mathbb{Z})^{3}$. 
Let $N$ be a positive integer, $\omega$ an integer of $k$ which is prime to $2 N$, and $r, s, r_{1}, s_{1} \in \frac{1}{N} \mathbb{Z}^{2}$. Then we have

$$
\Phi\left(z_{0} ; r, s ; r_{1}, s_{1}\right) \in k_{2 N^{2}}
$$

and

$$
\Phi\left(z_{0} ; r, s ; r_{1}, s_{1}\right)^{\left(\frac{k_{2 N 2} / k}{(\omega)}\right)}=\Phi^{R(\varphi(\omega))}\left(z_{0} ; r, s ; r_{1}, s_{1}\right)
$$

by Shimura's reciprocity law, where $\left(\frac{k_{2 N^{2}} / k}{(\omega)}\right)$ is the image of $(\omega)$ by Artin mapping (cf. [5, Proposition 2.2]). Moreover we have the following by [4, Propositions 1 and 2]:

Lemma. If $r, s, r_{1}, s_{1} \in \frac{1}{3} \mathbb{Z}^{2}$, then $\Phi\left(z_{0} ; r, s ; 0,0\right)$ is an algebraic integer of $K$ and $\Phi\left(z_{0} ; r, s ; r_{1}, s_{1}\right)^{3} \in k_{6}$.

Let $M$ be the subgroup of $k^{\times}$generated by integers of $k$ which are prime to 6 . We put $\tilde{M}=\{(\alpha) \mid \alpha \in M\}$. Then, by class field theory, we have

$$
G\left(k_{6} / k\right) \cong \tilde{M} / \tilde{S}_{6} \cong(M / U) /\left(S_{6} U / U\right) \cong M / S_{6} U \cong\left(M / S_{6}\right) /\left(S_{6} U / S_{6}\right) .
$$

Since

$$
M / S_{6} \cong\left(\mathfrak{O}_{k} / 6 \mathfrak{O}_{k}\right)^{\times} \cong\left(\mathfrak{O}_{k} / 2 \mathfrak{O}_{k}\right)^{\times} \omega\left(\mathfrak{O}_{k} / 3 \mathfrak{O}_{k}\right)^{\times},
$$

the order of $M / S_{6}$ is $15 \cdot 80$. We put $u=(-1+\sqrt{5}) / 2$; then we have $S_{6} U / S_{6}=$ $\left\langle u S_{6}, \zeta S_{6}\right\rangle$. Hence the order of $S_{6} U / S_{6}$ is 120 , because the order of $u S_{6}$ is 24 and the order of $\zeta S_{6}$ is 5 . Hence $\left[k_{6}: k\right]=10$. Moreover we have $\left\langle(-1-2 \zeta)+3 \mathfrak{O}_{k}\right\rangle=$ $\left(\mathfrak{O}_{k} / 3 \mathfrak{O}_{k}\right)^{\times}$and the order of $(3 \zeta+2)+2 \mathfrak{O}_{k}$ is 5 , which shows that the order of $(\zeta+2) S_{6}$ is 80 because $\zeta+2 \equiv 3 \zeta+2(\bmod 2)$ and $\zeta+2 \equiv-1-2 \zeta(\bmod 3)$. This shows that $M / U S_{6}=\left\langle(\zeta+2) U S_{6}\right\rangle$, because $(\zeta+2)^{8} \equiv-\zeta^{4}(\bmod 3)$ and $(\zeta+2)^{8} \equiv \zeta^{3}(\bmod 2)$. In a similar way, we see that $\left[k_{3}: k\right]=2$.

Now, we suppose $r, s \in \frac{1}{3} \mathbb{Z}^{2}$. We compute the norm of $\Phi\left(z_{0} ; r, s ; 0,0\right)^{3}$ from $k_{6}$ to $\mathbb{Q}$ by using the Lemma, (11), and [5, Proposition 1.3 ]. First, by (11), we have

$$
\begin{aligned}
N_{k_{6} / \mathbb{Q}(\sqrt{5})}\left(\Phi\left(z_{0} ; r, s ; 0,0\right)^{3}\right) & =\prod_{\nu=1}^{10}\left|\left(\Phi\left(z_{0} ; r, s ; 0,0\right)^{3}\right)^{\left(\frac{k_{6} / k}{(\zeta+2)^{\nu}}\right)}\right|^{2} \\
& =\left|\prod_{\nu=1}^{10}\left(\Phi^{R\left(\varphi(\zeta+2)^{\nu}\right)}\left(z_{0} ; r, s ; 0,0\right)^{3}\right)\right|^{2}
\end{aligned}
$$

Since

$$
R(\varphi(\zeta+2)) \equiv\left(\begin{array}{rrrr}
1 & 0 & 0 & 0 \\
0 & 1 & 0 & 0 \\
0 & 0 & 11 & 0 \\
0 & 0 & 0 & 11
\end{array}\right)\left(\begin{array}{rrrr}
3 & 0 & -1 & 1 \\
2 & 2 & 0 & -1 \\
15 & -10 & 10 & -5 \\
-10 & 5 & 5 & 20
\end{array}\right) \quad(\bmod 18)
$$

we can compute $\left|\Phi^{R\left(\varphi(\zeta+2)^{\nu}\right)}\left(z_{0} ; r, s ; 0,0\right)\right|^{2}$ by [5, Proposition 1.3]; for example,

$$
\left|\Phi^{R(\varphi(\zeta+2))}\left(z_{0} ;\left(\begin{array}{c}
\frac{1}{3} \\
0
\end{array}\right),\left(\begin{array}{l}
0 \\
0
\end{array}\right) ;\left(\begin{array}{l}
0 \\
0
\end{array}\right),\left(\begin{array}{l}
0 \\
0
\end{array}\right)\right)\right|^{2}=\left|\Phi\left(z_{0} ;\left(\begin{array}{c}
\frac{1}{2} \\
0
\end{array}\right),\left(\begin{array}{c}
\frac{2}{3} \\
\frac{5}{6}
\end{array}\right) ;\left(\begin{array}{c}
\frac{1}{2} \\
0
\end{array}\right),\left(\begin{array}{l}
0 \\
\frac{1}{2}
\end{array}\right)\right)\right|^{2} .
$$

Since $\Phi\left(z_{0} ; r, s ; 0,0\right)^{3}$ is an algebraic integer of $k_{6}$ and since the square of the absolute value of a conjugate of $\Phi\left(z_{0} ; r, s ; 0,0\right)$ over $\mathbb{Q}$ is of the form

$$
\left|\Phi\left(z_{0} ; r^{\prime}, s^{\prime} ; r^{\prime \prime}, s^{\prime \prime}\right)\right|^{2}
$$


for some $r^{\prime}, s^{\prime} \in \frac{1}{6} \mathbb{Z}^{2}$ and some $r^{\prime \prime}, s^{\prime \prime} \in \frac{1}{2} \mathbb{Z}^{2}$, it follows that we can determine $N_{k_{6} / \mathbb{Q}}\left(\Phi\left(z_{0} ; r, s ; 0,0\right)^{3}\right)$ with some luck by the method explained in the next section. As a result of computation, we have

$$
N_{k_{6} / \mathbb{Q}}\left(\Phi\left(z_{0} ;\left(\begin{array}{l}
\frac{1}{3} \\
0
\end{array}\right),\left(\begin{array}{l}
0 \\
0
\end{array}\right) ;\left(\begin{array}{l}
0 \\
0
\end{array}\right),\left(\begin{array}{l}
0 \\
0
\end{array}\right)\right)^{3}\right)=2^{48}
$$

and

$$
N_{k_{6} / \mathbb{Q}}\left(\Phi\left(z_{0} ;\left(\begin{array}{l}
\frac{1}{3} \\
\frac{2}{3}
\end{array}\right),\left(\begin{array}{l}
\frac{1}{3} \\
0
\end{array}\right) ;\left(\begin{array}{l}
0 \\
0
\end{array}\right),\left(\begin{array}{l}
0 \\
0
\end{array}\right)\right)^{3}\right)=2^{48}
$$

Since the order of $2+5 \mathbb{Z}$ is 4 , the ideal $2 \mathfrak{O}_{k}$ is a prime ideal of $\mathfrak{O}_{k}$. Moreover, the ideal $2 \mathfrak{O}_{k}$ splits in $k_{3} / k$ since $-2 \equiv 1(\bmod 3)$. Hence the decomposition group of $2 \mathfrak{O}_{k}$ with respect to $k_{6} / k$ is $\left\langle\left(\frac{k_{6} / k}{(2+\zeta)}\right)^{2}\right\rangle$, because $2 \mathfrak{O}_{k}$ ramifies in $k_{6} / k$. In view of (21) and (3), this shows that $\varepsilon_{\nu}^{3}$ and $\eta_{\nu}^{3}$ are units in $k_{6}$ for all $\nu \in \mathbb{Z}$. Now, noting that $\zeta_{3}^{R\left(\varphi(2+\zeta)^{2}\right)}=\zeta_{3}$, we have

$$
\begin{gathered}
\left(\frac{\Phi^{R\left(\varphi(2+\zeta)^{\nu}\right)}\left(z_{0} ; r, s ; 0,0\right)}{\Phi^{R\left(\varphi(2+\zeta)^{\nu+2}\right)}\left(z_{0} ; r, s ; 0,0\right)}\right)^{\left(\frac{k_{18} / k}{\left(\omega_{i}\right)}\right)} \\
=\frac{\Phi^{R\left(\varphi\left(\omega_{i}\right)\right) R\left(\varphi(2+\zeta)^{\nu}\right)}\left(z_{0} ; r, s ; 0,0\right)}{\Phi^{R\left(\varphi\left(\omega_{i}\right)\right) R\left(\varphi(2+\zeta)^{\nu}\right) R\left(\varphi(2+\zeta)^{2}\right)}\left(z_{0} ; r, s ; 0,0\right)} \\
=\frac{\Phi^{R\left(\varphi(2+\zeta)^{\nu}\right)}\left(z_{0} ; r, s ; 0,0\right)}{\Phi^{R\left(\varphi(2+\zeta)^{\nu+2}\right)}\left(z_{0} ; r, s ; 0,0\right)}
\end{gathered}
$$

which shows that $\varepsilon_{\nu}$ and $\eta_{\nu}$ are contained in $k_{6}$. Hence $\varepsilon_{\nu}$ and $\eta_{\nu}$ are units in $k_{6}$. It is clear that $\rho_{0}, \rho_{1}$ and $\rho_{2}$ are units in $k_{6}$, because $\zeta_{3}, \zeta_{5} \in k_{6}$.

Let $\tau=\left(\frac{k_{6} / k}{(\zeta+2)}\right) \in G\left(k_{6} / k\right)$. Remember that $\sigma$ is the element of $G(k / \mathbb{Q})$ defined by $\zeta^{\sigma}=\zeta^{2}$. We extend $\sigma$ to $k_{6}$ and keep the notation for simplicity. Then, as we explain in the next section, we see that the rank of the matrix

$$
\left(\log \left|u^{g}\right|\right)
$$

is 19 , where $u$ runs over $\left\{\varepsilon_{0}, \cdots, \varepsilon_{7}, \eta_{0}, \cdots, \eta_{7}, \rho_{0}, \rho_{1}, \rho_{2}\right\}$ and $g$ is of the form $\sigma^{i} \tau^{j}(0 \leq i \leq 1,0 \leq j \leq 9)$. This completes the proof of theorem.

\section{Computation}

In this section, we explain how we computed (2), (3) and the rank of (4). Since theta series converges rapidly, one can easily compute an approximate value of it with high precision. In our case, a rough estimate

$$
\begin{array}{r}
\left|\Theta\left(0, z_{0} ; r^{\prime}, s^{\prime}\right)-\sum_{\substack{x \in \mathbb{Z}^{2} \\
|x|^{2}<n}} e\left(\frac{1}{2} t\left(x+r^{\prime}\right) z_{0}\left(x+r^{\prime}\right)+{ }^{t}\left(x+r^{\prime}\right) s^{\prime}\right)\right| \\
<\frac{4(n+1-n \exp (-2.8 \pi / 180)) \exp (-2.8 n \pi / 180)}{(1-\exp (-2.8 \pi / 180))^{2}}
\end{array}
$$


was enough for computing the approximate value of $\left|\Phi\left(z_{0} ; r^{\prime}, s^{\prime} ; r^{\prime \prime}, s^{\prime \prime}\right)\right|$ with a precision of 200 digits. We computed the approximate values of

$$
N_{k_{6} / \mathbb{Q}(\sqrt{5})}\left(\Phi\left(z_{0} ;\left(\begin{array}{l}
\frac{1}{3} \\
0
\end{array}\right),\left(\begin{array}{l}
0 \\
0
\end{array}\right) ;\left(\begin{array}{l}
0 \\
0
\end{array}\right),\left(\begin{array}{l}
0 \\
0
\end{array}\right)\right)^{3}\right) \cdot N_{k_{6} / \mathbb{Q}(\sqrt{5})}\left(\Phi\left(z_{0} ; r^{\prime}, s^{\prime} ; r^{\prime \prime}, s^{\prime \prime}\right)^{3}\right)
$$

for all $r^{\prime}, s^{\prime} \in \frac{1}{6} \mathbb{Z}^{2}$ and $r^{\prime \prime}, s^{\prime \prime} \in \frac{1}{2} \mathbb{Z}^{2}$, and found that $2^{48}$ is the only possible integral value for (5). Hence we have (2). Similarly we have (3).

Next we consider (44). The actions of $\sigma$ and $\tau$ on $\rho_{i}$ are given by $\zeta_{5}^{\sigma}=\zeta_{5}^{2}, \zeta_{3}^{\sigma}=\zeta_{3}$ and $\zeta_{5}^{\tau}=\zeta_{5}, \zeta_{3}^{\tau}=\zeta_{3}^{2}$. Namely, $\rho_{1}=\rho_{0}^{\sigma}$ and $\rho_{2}=\rho_{0}^{\tau}$. Moreover, using $r^{\prime}, s^{\prime} \in \frac{1}{6} \mathbb{Z}^{2}$ and $r^{\prime \prime}, s^{\prime \prime} \in \frac{1}{2} \mathbb{Z}^{2}$ for which the values of (5D) are near $2^{48}$, we found ten possible values of $\log \left|\varepsilon_{0}^{\sigma}\right|$. All the values are derived from one by actions of $\tau$. So we may regard any of these as $\log \left|\varepsilon_{0}^{\sigma}\right|$ by replacing $\sigma$ with $\sigma \tau^{i}$. Since $\sigma^{-1} \tau \sigma=\tau^{7}$ and $\varepsilon_{i}^{\tau}=\varepsilon_{i+1}$, we calculate $\log \left|\varepsilon_{i}^{\sigma \tau^{j}}\right|$ from

$$
\varepsilon_{i}^{\sigma \tau^{j}}=\varepsilon_{0}^{\tau^{i} \sigma \tau^{j}}=\varepsilon_{0}^{\sigma \sigma^{-1} \tau^{i} \sigma \tau^{j}}=\left(\varepsilon_{0}^{\sigma}\right)^{\tau^{7 i+j}}
$$

Similarly we have ten possible values of $\log \left|\eta_{0}^{\sigma}\right|$. But this time, we could not specify the value of $\log \left|\eta_{0}^{\sigma}\right|$. We calculated the rank of (4) for all possible values of $\log \left|\eta_{0}^{\sigma}\right|$ and verified that the rank is 19 for all cases. Hence we conclude that the rank of (4) is 19.

A 64-bit work station DEC Alpha 500/333 needed eight hours for these computations.

\section{REFERENCES}

[1] J. Arledge, S-units attached to genus 3 hyperelliptic curves, J. Number Theory 63 (1997), 12-29. MR 98c: 11057

[2] J. Boxall and E. Bavencoffe, Quelques propriétés arithmétiques des points de 3-division de la jacobienne de $y^{2}=x^{5}-1$, Sém. Théor. Bordeaux 4-1 (1992), 113-128. MR 93m:11051

[3] D. Grant, Units from 3- and 4-torsion on Jacobians of curves of genus 2, Compositio Math. 94 (1994), 311-320. MR 95j:11053

[4] K. Komatsu, Construction of normal basis by special values of Siegel modular functions, submitted to Proc. Amer. Math. Soc.

[5] G. Shimura, Theta functions with complex multiplication, Duke Math. J., 43 (1976), 673-696. MR 54:12664

Department of Mathematics, College of Industrial Technology, Nihon University, 2-11-1 Shin-ei, Narashino, Chiba, Japan

E-mail address: fukuda@math.cit.nihon-u.ac.jp

Department of Information and Computer Science, School of Science and EngineerIng, Waseda University, 3-4-1 Okubo, Shinjuku, Tokyo 169, Japan

E-mail address: kkomatsu@mn.waseda.mse.jp 